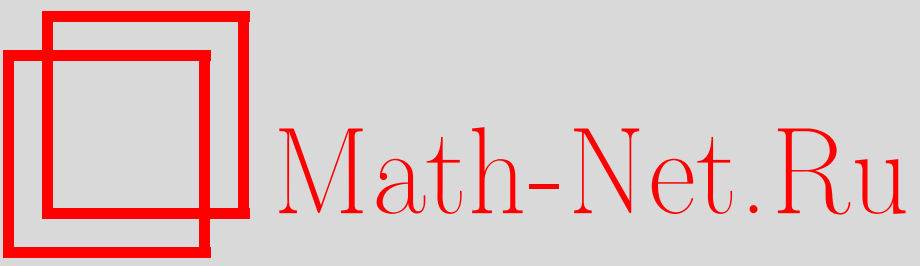

В. В. Стружанов, Е. А. Бахарева, Итерационные процедуры расчёта параметров равновесия и устойчивость процесса чистого изгиба балок из пластических и хрупких разупрочняющихся материалов, Вестн. Сам. гос. техн. ун-та. Сер. Физ.-мат. науки, 2010, выпуск 1(), 84-95

DOI: https://doi.org/10.14498/vsgtu784

Использование Общероссийского математического портала Math-Net.Ru подразумевает, что вы прочитали и согласны с пользовательским соглашением http://www . mathnet.ru/rus/agreement

Параметры загрузки:

IP : 18.208 .226 .222

26 апреля 2023 г., 14:05:08 
УДК 539.3

\title{
ИТЕРАЦИОННЫЕ ПРОЦЕДУРЫ РАСЧЁТА ПАРАМЕТРОВ РАВНОВЕСИЯ И УСТОЙЧИВОСТЬ ПРОЦЕССА ЧИСТОГО ИЗГИБА БАЛОК ИЗ ПЛАСТИЧНЫХ И ХРУПКИХ РАЗУПРОЧНЯЮЩИХСЯ МАТЕРИАЛОВ
}

\section{В. В. Стружнанов, Е. А. Бахарева}

1 Институт машиноведения УрО РАН,

620219, г. Екатеринбург, ул. Комсомольская, 34

2 Уральский государственный университет им. А. М. Горького, 620219, г. Екатеринбург, пр-т Ленина, 51.

E-mails: stru@imach.uran.ru, bahareva.e.a@mail.ru

\begin{abstract}
Приведены итераиионные процедуры расчёта напряжённо-деформированного состояния при чистом изгибе балок прямоугольного поперечного сечения, выполненных из пластичных и хрупких материалов, обладаюших эффектом деформационного разупрочнения. Исследована сходимость методов и установлена свлзь между расходимостью итераций и устойчивостью процесса нагружения.
\end{abstract}

Ключевые слова: чистый изгиб, жёсткое и мягкое нагружения, итерационная процедура, сходимость, устойчивость, метод простой итерации.

Введение. Итерационные схемы, предложенные в работах $[1,2]$ для решения задач по определению напряжённо-деформированного состояния некоторых элементов конструкций при учёте деформационного разупрочнения материалов, применены для расчётов параметров равновесия прямоугольных балок из упругопластических, партипластических и упругохрупких материалов с разупрочнением. Балки подвергаются чистому изгибу по мягкой и жёсткой схемам нагружения. Исследована сходимость итераций и установлена связь между началом расходимости последовательных приближений и моментом потери устойчивости процесса деформирования балок.

1. Определяющие соотношения. Рассмотрим чистый изгиб достаточно длинной балки с прямоугольным поперечным сечением. Высота и ширина балки соответственно равны $2 h$ и $b$. Деформирование осуществляем квазистатически при неизменной температуре, либо задавая монотонно возрастающий изгибающий момент $M$ (мягкое нагружение), либо монотонно увеличивая кривизну балки $\kappa$ (жёсткое нагружение). Свойства материала определяет полная диаграмма растяжения $\sigma_{\varepsilon}(\varepsilon)$. Она состоит из восходящей (упрочнение) и нисходящей (разупрочнение) до нуля ветвей. Символами $\sigma_{\varepsilon}^{B}$ и $\varepsilon^{B}$ обозначены соответственно напряжение предела прочности и отвечающая ему деформация растяжения, $\varepsilon^{Z}$ - деформация разрушения (рис. 1). Функцию $\sigma_{\varepsilon}$ полагаем однозначной и непрерывно дифференцируемой.

В случае чистого изгиба единственной ненулевой компонентой тензора напряжений является продольное напряжение $\sigma_{x}=\sigma(y)$, а продольная деформация $\varepsilon_{x}=\varepsilon(y)$ линейно распределена по высоте балки (рис. 2). Считаем, что диаграмма при сжатии симметрична относительно начала координат

Валерий Владимирович Стружанов (д.ф.-м.н., проф.), главный научный сотрудник, лаб. микромеханики материалов. Елена Александровна Бахарева, магистрант, каф. теоретической механики. 
диаграмме растяжения. Следовательно, положение нейтральной плоскости, в точках которой продольные напряжение и деформация равны нулю, неизменно. Отсюда функции $\sigma(y)$ и $\varepsilon(y)$ являются нечетными.

Наклон диаграмм при сжатии и растяжении характеризует четная функция $E^{p}(\varepsilon)=d \sigma_{\varepsilon} / d \varepsilon$, имеющая смысл касательного модуля. Её знак определяет состояние материала. Если $E^{p}>0$, то материал находится в состоянии упрочнения, если $E^{p}<0$, то в состоянии разупрочнения.

Разгрузка происходит по линейному закону. В общем случае (партипластический материал) модуль разгрузки равен $E^{u}=\sigma /\left(\varepsilon-\varepsilon^{p}\right)\left(E^{s} \leqslant E^{u} \leqslant E\right)$. Здесь $E$ - модуль упругости (модуль Юнга), $E^{s}-$ секущий модуль, $\varepsilon^{p}-$ остаточная пластическая деформация. Полная деформация представляется суммой $\varepsilon=\varepsilon^{e}+\varepsilon^{p}$, где $\varepsilon^{e}, \varepsilon^{p}$ - соответственно упругая и пластическая составляющие полной деформации. В предельных случаях $E^{u}=E$ (упругопластический материал) и $E^{u}=E^{s}$ (упругохрупкий материал) (рис. 1).

Для партипластического материала (частично пластического) при одноосном растяжении соотношение, связывающее напряжение и деформацию, имеет вид

$$
\sigma=E^{u} \varepsilon^{e}=E^{u}\left(\varepsilon-\varepsilon^{p}\right) .
$$

Дифференциал от выражения (1)

$$
d \sigma=d E^{u}\left(\varepsilon-\varepsilon^{p}\right)+E^{u}\left(d \varepsilon-d \varepsilon^{p}\right) .
$$

С другой стороны, справедливо равенство

$$
d \sigma=E^{p} d \varepsilon .
$$

Приравнивая выражения (2) и (3), после необходимых преобразований получаем уравнение

$$
\frac{d \varepsilon^{p}}{d \varepsilon}=1-\frac{E^{p}(\varepsilon)}{E^{u}(\varepsilon)}+\frac{\left(\varepsilon-\varepsilon^{p}(\varepsilon)\right)}{E^{u}(\varepsilon)} \frac{d E^{u}}{d \varepsilon},
$$

которое можно записать в виде

$$
d\left(\varepsilon^{p} E^{u}\right)=d\left(\varepsilon E^{u}\right)-E^{p} d \varepsilon .
$$

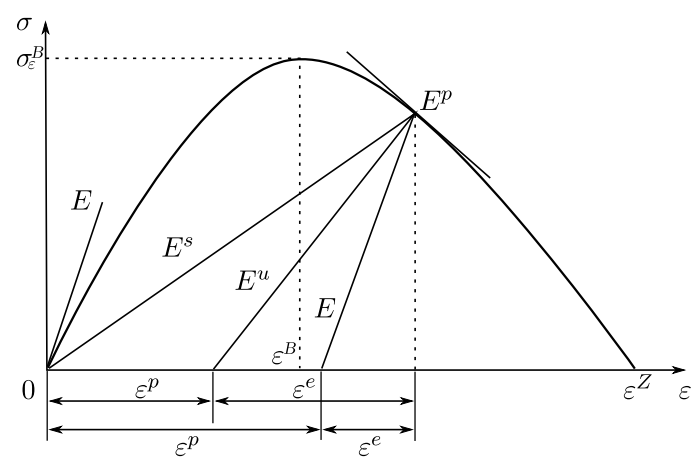

Рис. 1. Полная диаграмма деформирования при растяжении и модули разгрузки для различных типов материалов

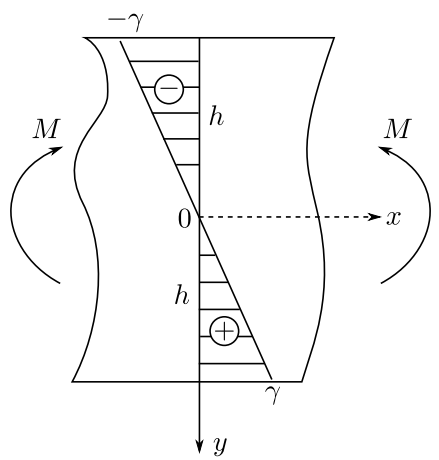

Рис. 2. Распределение продольных деформаций; $\gamma=\varepsilon(h)$ - деформация наиболее растянутых волокон балки 
Интегрируя равенство (5), находим выражение

$$
\varepsilon^{p}=\varepsilon-\frac{1}{E^{u}(\varepsilon)} \int_{0}^{\varepsilon} E^{p} d \varepsilon
$$

определяющее пластические деформации в партипластическом материале.

2. Краевые задачи (мягкое нагружение). Очевидно, что напряжения и деформации при чистом изгибе тождественно удовлетворяют дифференциальным уравнениям равновесия и условиям совместности. Следовательно, требуется найти только такое их распределение по высоте балки, при котором в каждой точке перпендикулярного срединной плоскости сечения справедливо соотношение (1) при значениях модулей $E^{u}(\varepsilon)$, вычисленных для величин полных деформаций с использованием диаграммы деформирования, и пластических деформаций $\varepsilon^{p}$, определенных по формуле (6). При выполнении данных условий $\sigma(y) \in \sigma_{\varepsilon}$. Кроме того, должны выполняться равенства

$$
\int_{-h}^{h} \sigma d y=0, \quad b \int_{-h}^{h} \sigma y d y=2 b \int_{0}^{h} \sigma y d y=M
$$

(граничные условия). Выражения (7), где первое равенство удовлетворяется тождественно в силу нечетности функции $\sigma(y)$, играют также роль статических уравнений равновесия.

Отметим, что изгибающий момент можно представить в виде разности

$$
M=2 b \int_{0}^{h} E^{u}\left(\varepsilon-\varepsilon^{p}\right) y d y=\kappa I^{u}-M^{\phi} \quad(\varepsilon=\kappa y),
$$

где

$$
I^{u}=I^{u}(\kappa)=2 b \int_{0}^{h} E^{u}(\kappa y) y^{2} d y
$$

- жёсткость при изгибе упругой балки с модулем $E^{u}(\varepsilon)$;

$$
M^{\phi}=M^{\phi}(\kappa)=2 b \int_{0}^{h} E^{u}(\kappa y) \varepsilon^{p}(\kappa y) y d y
$$

- фиктивный изгибающий момент, определяемый псевдонапряжениями $\sigma^{*}=$ $=E \varepsilon^{p}$.

Исходя из разбиения (8) решение исходной краевой задачи можно представить в виде суммы решений задач $(\mathrm{A})$ и $(\mathrm{B})$. Задача $(\mathrm{A})$ - это задача об определении напряжений и деформаций, возникающих в упругой балке с модулями $E^{u}=E^{u}(\kappa y)$. Её решение дают выражения

$$
\kappa^{\prime}=M / I^{u}, \quad \varepsilon^{\prime}=\kappa^{\prime} y, \quad \sigma^{\prime}=E^{u}(\kappa y) \varepsilon^{\prime} .
$$

Ясно, что

$$
2 b \int_{0}^{h} \sigma^{\prime} y d y=2 b \int_{0}^{h} E^{u}(\kappa y) \frac{M}{I^{u}} y^{2} d y=\frac{M}{I^{u}} 2 b \int_{0}^{h} E^{u}(\kappa y) y^{2} d y=M,
$$

то есть напряжения $\sigma^{\prime}(y)$ удовлетворяют условию (7). 
Задача (В) формулируется следующим образом. Мысленно разгрузим балку. После разгрузки в ней остаются только пластические деформации, инициируюшие появление (так называемых) остаточных напряжений, которые самоуравновешиваются при отсутствии внешней нагрузки. В этом случае напряжённо-деформированное состояние определяют равенства

$$
\kappa^{\prime \prime}=M^{\phi} / I^{u}, \quad e^{\prime}=\kappa^{\prime \prime} y, \quad \sigma^{\prime \prime}=E^{u}(\kappa y)\left(e^{\prime}-\varepsilon^{p}\right),
$$

где $\kappa^{\prime \prime}$ - кривизна балки, появляющаяся из-за наличия в ней остаточных напряжений, $e^{\prime}$ - линейно распределенная деформация, отвечающая данной кривизне и фиксируемая сторонним наблюдателем (внешним по отношению к балке). Ясно, что

$$
\begin{aligned}
2 b \int_{0}^{h} \sigma^{\prime \prime} y d y=2 b\left(\int_{0}^{h} E^{u}(\kappa y) \frac{M^{\phi}}{I^{u}} y^{2} d y-\int_{0}^{h} E^{u}(\kappa y) \varepsilon^{p}(\kappa y) y d y\right)= \\
=2 b\left(\frac{M^{\phi}}{I^{u}} \cdot I^{u}-M^{\phi}\right)=0 .
\end{aligned}
$$

3. Итерационный процесс (мягкое нагружение). Пусть при изгибающем моменте $M_{0}$ балка имеет кривизну $\kappa_{0}$ и находится в состоянии равновесии, параметры которого равны $E^{u}=E_{0}^{u}\left(\varepsilon_{0}\right), \sigma=\sigma_{0}\left(\varepsilon_{0}\right)=\sigma_{\varepsilon}\left(\varepsilon_{0}\right), \varepsilon=\varepsilon_{0}=$ $=\kappa_{0} y, \varepsilon^{p}=\varepsilon_{0}^{p}\left(\varepsilon_{0}\right)$. Возмутим это равновесие, увеличив изгибающий момент на величину $M_{\Delta}$. Параметры возмущения найдем, используя решение задачи (А), по формулам (9). Имеем

$$
\kappa_{\Delta}^{\prime}=M_{\Delta} / I_{0}^{u}, \quad \varepsilon_{\Delta}^{\prime}=\kappa_{\Delta}^{\prime} y, \quad \sigma_{\Delta}^{\prime}=E_{0}^{u} \varepsilon_{\Delta}^{\prime} .
$$

Здесь $I_{0}^{u}=I^{u}\left(\kappa_{0}\right)$. Тогда параметры балки в возмущенном состоянии находятся как

$$
\kappa_{1}=\kappa_{0}+\kappa_{\Delta}^{\prime}=M / I_{0}^{u}, \quad \varepsilon_{1}=\varepsilon_{0}+\varepsilon_{\Delta}^{\prime}=\kappa_{1} y, \quad \sigma_{1}=\sigma_{0}+\sigma_{\Delta}^{\prime},
$$

где $M=M_{0}+M_{\Delta}$.

Данные величины соответствуют равновесному состоянию при неизменившихся значениях $E_{0}^{u}$ и $\varepsilon_{0}^{u}$. Отсюда $\sigma_{1}(y) \notin \sigma_{\varepsilon}$. Следовательно, величины модулей и пластических деформаций должны поменяться и отвечать деформации $\varepsilon_{1}$ в соответствии с полной диаграммой деформирования. Поэтому модули станут равными $E_{1}^{u}=E^{u}\left(\varepsilon_{1}\right)$, а пластические деформации достигнут величины $\varepsilon_{1}^{p}$, определяемой по формуле (6), в которой полагаем $E^{u}=E_{1}^{u}$, $\varepsilon=\varepsilon_{1}$

Таким образом, найденное равновесие нарушается, и балка под действием момента $M$ должна перейти в другое равновесное состояние, параметры которого являются суммой решений задач $(\mathrm{A})$ и $(\mathrm{B})$ для балки с модулями $E_{1}^{u}$ и пластическими деформациями $\varepsilon_{1}^{p}$.

Используя формулы (9) и (10), находим

$\kappa_{1}^{\prime}=M / I_{1}^{u}, \quad \varepsilon_{1}^{\prime}=\kappa_{1}^{\prime} y, \quad \sigma_{1}^{\prime}=E_{1}^{u} \varepsilon_{1}^{\prime}, \quad \kappa_{1}^{\prime \prime}=M_{1}^{\phi} / I_{1}^{u}, e_{1}^{\prime}=\kappa_{1}^{\prime \prime} y, \quad \sigma_{1}^{\prime \prime}=E_{1}^{u}\left(e_{1}^{\prime}-\varepsilon_{1}^{p}\right)$. 
Здесь $I_{1}^{u}=I^{u}\left(\kappa_{1}\right)$ - новая жёсткость балки с модулями упругости $E_{1}^{u}\left(\kappa_{1} y\right)$, $M_{1}^{\phi}=M^{\phi}\left(\kappa_{1}\right)$ - новое значение фиктивного момента. Скорректированное напряжённо-деформированное состояние определяется суммой задач (А) и (B), а именно

$$
\kappa_{2}=\kappa_{1}^{\prime}+\kappa_{1}^{\prime \prime}, \quad \varepsilon_{2}=\varepsilon_{1}^{\prime}+e_{1}^{\prime}=\kappa_{2} y, \quad \sigma_{2}=\sigma_{1}^{\prime}+\sigma_{1}^{\prime \prime} .
$$

Если снова $\sigma_{2}(y) \notin \sigma_{\varepsilon}$, то выражения (11) будем считать первым приближением к параметрам искомого равновесного состояния и проведем следующую корректировку. Находим $E_{2}^{u}=E^{u}\left(\varepsilon_{2}\right)$ и по формуле $(6)$, где уже $\varepsilon=\varepsilon_{2}$ и $E^{u}=E_{2}^{u}$, вычисляем новые значения пластических деформаций $\varepsilon_{2}^{p}(y)$. Для параметров $E_{2}^{u}$ и $\varepsilon_{2}^{p}$ ищем решения задач $(\mathrm{A})$ и (В) и, складывая их, получаем второе приближение. Процесс повторяем необходимое число раз. Схематически итерационная процедура показана на рис. 3 для материального элемента и на рис. 4 для балки в целом.

На $n$-ном шаге итерационного процесса получаем равенство

$$
\kappa_{n+1}=\left(M+M_{n}^{\phi}\right) / I_{n}^{u}=\varphi\left(\kappa_{n}\right)
$$

где величины $I_{n}^{u}=I^{u}\left(\kappa_{n}\right), M_{n}^{\phi}=M^{\phi}\left(\kappa_{n}\right)$ подсчитаны для функций $\varepsilon_{n}=$ $=\kappa_{n} y, E_{n}^{u}=E^{u}\left(\kappa_{n} y\right), \varepsilon_{n}^{p}=\varepsilon^{p}\left(\kappa_{n} y\right)$. Формула (12) определяет метод простой итерации, который сходится, если $\sup \left|\frac{d \varphi}{d \kappa}\right|<1$ (см. [3]). Здесь функция $\varphi(\kappa)=$ $=\left(M+M^{\phi}(\kappa)\right) / I^{u}(\kappa)$, причём функции $I^{u}(\kappa)$ и $M^{\phi}(\kappa)$ таковы, что

$$
\kappa I^{u}-M^{\phi}=M=\text { const, }
$$

то есть функции $I^{u}$ и $M^{\phi}$ изменяются, а указанная разность остается постоянной. Это утверждение справедливо потому, что на каждом итерационном

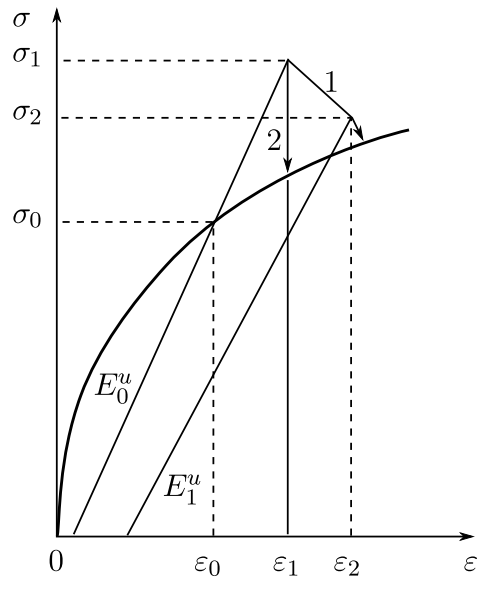

Рис. 3. Схема итерационных процессов для элемента материала: 1 - мягкое догружение, 2 - жёсткое догружение

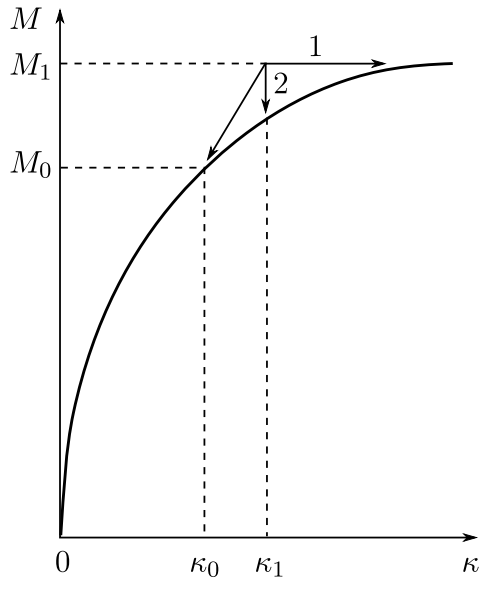

Рис. 4. Схема итерационных процессов для балки: 1 - мягкое догружение, 2 жёсткое догружение 
шаге вычисляются параметры некоторого равновесия, отвечающие заданному моменту $M$, то есть в ходе итераций балка как-бы переходит из одного равновесия в другое, стремясь к положению реального равновесия.

Найдём производную от функции $\varphi(\kappa)$. В силу условия равновесия (8), используя соотношение (4), имеем

$$
\frac{d \varphi}{d \kappa}=\frac{I^{u} \frac{d M^{\phi}}{d \kappa}-\left(M+M^{\phi}\right) \frac{d I^{u}}{d \kappa}}{\left(I^{u}\right)^{2}}=\frac{I^{u} \frac{d M^{\phi}}{d \kappa}-\kappa I^{u} \frac{d I^{u}}{d \kappa}}{\left(I^{u}\right)^{2}}
$$

где

$$
\begin{gathered}
\frac{d M^{\phi}}{d \kappa}=2 b\left(\int_{0}^{h} \frac{d E^{u}}{d \kappa} \varepsilon^{p} y d y+\int_{0}^{h} E^{u} \frac{d \varepsilon^{p}}{d \kappa} y d y\right), \quad \frac{d I^{u}}{d \kappa}=2 b \int_{0}^{h} \frac{d E^{u}}{d \kappa} y^{2} d y, \\
\frac{d E^{u}}{d \kappa}=\frac{d E^{u}}{d \varepsilon} \frac{d \varepsilon}{d \kappa}=\frac{d E^{u}}{d \varepsilon} y \quad(\varepsilon=\kappa y), \\
\frac{d \varepsilon^{p}}{d \kappa}=\frac{d \varepsilon^{p}}{d \varepsilon} \frac{d \varepsilon}{d \kappa}=\left(1-\frac{E^{p}}{E^{u}}+\frac{\left(\varepsilon-\varepsilon^{p}\right)}{E^{u}} \frac{d E^{u}}{d \varepsilon}\right) y .
\end{gathered}
$$

Тогда

$$
\begin{aligned}
& \frac{1}{2 b}\left(\frac{d M^{\phi}}{d \kappa}-\kappa \frac{d I^{u}}{d \kappa}\right)=\int_{0}^{h} \frac{d E^{u}}{d \varepsilon} \varepsilon^{p} y^{2} d y+\int_{0}^{h} E^{u} y^{2} d y-\int_{0}^{h} E^{p} y^{2} d y+ \\
& \quad+\int_{0}^{h} \frac{d E^{u}}{d \varepsilon} \varepsilon y^{2} d y-\int_{0}^{h} \frac{d E^{u}}{d \varepsilon} \varepsilon^{p} y^{2} d y-\kappa \int_{0}^{h} \frac{d E^{u}}{d \varepsilon} y^{3} d y=\int_{0}^{h} E^{u} y^{2} d y- \\
& -\int_{0}^{h} E^{p} y^{2} d y+\int_{0}^{h} \frac{d E^{u}}{d \varepsilon} \varepsilon y^{2} d y-\int_{0}^{h} \frac{d E^{u}}{d \varepsilon} \varepsilon y^{2} d y=\int_{0}^{h} E^{u} y^{2} d y-\int_{0}^{h} E^{p} y^{2} d y
\end{aligned}
$$

и, наконец,

$$
\frac{d \varphi}{d \kappa}=1-\frac{2 b \int_{0}^{h} E^{p} y^{2} d y}{2 b \int_{0}^{h} E^{u} y^{2} d y}=1-\frac{2 b I^{p}}{I^{u}} .
$$

Здесь величина $I^{u}$ всегда больше нуля, так как соответствующее подынтегральное выражение положительно. Поэтому процесс сходится, когда $I^{p}>0$ $\left(\frac{d \varphi}{d \kappa}<1\right)$, и расходится, когда $I^{p}<0\left(\frac{d \varphi}{d \kappa}>1\right)$.

Обращение интеграла $I^{p}$ в нуль, очевидно, является необходимым условием для расходимости итерационного процесса. Покажем, что это условие является и достаточным. Вычислим интеграл $I^{p}$ методом интегрирования по частям, используя следующее равенство:

$$
\int E^{p} d y=\int \frac{1}{\kappa} E^{p}(\kappa y) \kappa d y=\int \frac{1}{\kappa} \frac{d \sigma(\varepsilon)}{d \varepsilon} \frac{d \varepsilon}{d y} d y=\frac{1}{\kappa} \sigma(\kappa y) .
$$

Тогда 


$$
\begin{aligned}
I^{p}=\int_{0}^{h} E^{p} y^{2} d y= & \left.y^{2} \frac{\sigma(\kappa y)}{\kappa}\right|_{0} ^{h}- \\
& -2 \int_{0}^{h} \frac{\sigma(y)}{\kappa} y d y=\frac{h^{2}}{\kappa} \sigma(\kappa h)-\frac{2}{\kappa} \frac{M}{2 b}=\frac{1}{b \kappa}\left(b h^{2} \sigma(\gamma)-M\right),
\end{aligned}
$$

где

$$
\gamma=\kappa h, \quad M=2 b \int_{0}^{h} \sigma(y) y d y .
$$

Ясно, что уравнение $b h^{2} \sigma(\gamma)-M=0$ имеет только одно решение. Причём оно существует только тогда, когда в наиболее растянутых (сжатых) областях балки касательный модуль $E^{p}<0$ (в противном случае подинтегральное выражение положительно и $\left.I^{p}>0\right)$. Следовательно, если на каком-либо шаге итерационного процесса интеграл $I^{p}$ обращается в нуль, то при последующих итерациях напряжение $\sigma(\gamma)$ может разве лишь уменьшиться. Отсюда на всех последующих итерациях интеграл $I^{p}<0$. Таким образом, для начала расходимости итерационного процесса необходим и достаточным условием является обращение в нуль интеграла $I^{p}$.

Отметим, что выражение $2 b I^{p}$ определяет тангенциальную (мгновенную) изгибную жёсткость балки, в то время как выражение для $I^{u}$ есть жёсткость при изгибе упругой балки, модули которой равны модулям разгрузки.

Наконец, вычисляя производную от функции $M(\kappa)$, находим

$$
\frac{d M}{d \kappa}=2 b \int_{0}^{h} \frac{\partial \sigma}{\partial(\kappa y)} \frac{\partial(\kappa y)}{\partial \kappa} y d y=2 b \int_{0}^{h} E^{p}(\kappa y) y^{2} d y=2 b I^{p} .
$$

Следовательно, в наивысшей точке диаграммы $M=M(\kappa)$ выполняется равенство $I^{p}=0$, на восходящей ветви имеем $I^{p}>0$, на ниспадающей $-I^{p}<0$. Отсюда, постепенно увеличивая изгибающий момент с помощью изложенного итерационного процесса, возможно построить только восходящую ветвь диаграммы $M \sim \kappa$.

4. Устойчивость процесса деформирования балки (мягкое нагружение). Для определения устойчивости равновесия балки используем аппарат теории катастроф [4], опираясь на то, что каждый элемент находится в состоянии активного деформирования. В этом случае любой материал неотличим от нелинейно упругого материала, и поэтому существует потенциал напряжений в виде работы деформаций материального элемента, а именно

$$
F(\varepsilon)=\int_{0}^{\varepsilon} \sigma(e) d e .
$$

Очевидно, что функция $F$ чётная. Считая теперь изгибающий момент $M$ параметром управления, а кривизну $\kappa$ балки - параметром состояния, запишем выражение для полной энергии:

$$
\Pi(M, \kappa)=2 b \int_{0}^{h} F(\kappa y) d y-\int_{0}^{\kappa} M d \kappa \quad(\varepsilon=\kappa y) .
$$

Эта потенциальная функция задает однопараметрическое семейство функций параметров состояния. 
Критические точки функции П определяет уравнение

$$
\frac{\partial \Pi}{\partial \kappa}=2 b \int_{0}^{h} \sigma(\kappa y) y d y-M=0,
$$

связывающее параметры управления и состояния в положениях равновесия балки (устойчивых или неустойчивых). Вырожденные критические точки, в которых происходит смена типа равновесия, находятся из совместного решения уравнения (14) и уравнения

$$
\frac{\partial^{2} \Pi}{\partial \kappa^{2}}=2 b \int_{0}^{h} \frac{\partial \sigma}{\partial \varepsilon} \frac{\partial \varepsilon}{\partial \kappa} y d y=2 b \int_{0}^{h} E^{p} y^{2} d y=2 b I^{p}=0 .
$$

В начале процесса нагружения напряжённо-деформированное состояние балки устойчиво. В каждой точке $E^{p}>0$ и интеграл $I^{p}>0$. Поэтому при постепенном возрастании изгибающего момента согласно принципу промедления [4] балка будет сохранять устойчивое равновесие до тех пор, пока интеграл $I^{p}$ не обратится в нуль. В этот момент критическая точка, отвечающая данному положению равновесия, становится вырожденной и, следовательно, процесс нагружения балки теряет устойчивость. Очевидно, что за этим следует её разрушение. Но это же условие $\left(I^{p}=0\right)$ определяет и начало расходимости итерационного процесса. Таким образом, начало расходимости итераций совпадает с моментом потери устойчивости процесса деформирования балки.

Отметим, что потеря устойчивости процесса нагружения реализуется тогда, когда величина изгибающего момента превышает максимальное значение момента на диаграмме $M \sim \kappa$.

5. Итерационный процесс (жёсткое нагружение). При жёстком нагружении, фиксируя величину кривизны $\kappa$, сразу можно определить распределение деформаций по высоте балки $(\varepsilon=\kappa y)$. Затем, используя диаграмму деформирования, находим распределение модуля $E^{u}(y)$ и по формуле (6) - распределение пластических деформаций $\varepsilon^{p}(y)$. Наконец, вычисляем распределение напряжений по формуле (1) и по формуле (8) рассчитываем величину изгибающего момента, отвечающую заданной кривизне.

$\mathrm{C}$ другой стороны, данное напряжённо-деформированное состояние можно представить суммой решений двух задач $(\mathrm{C})$ и $(\mathrm{D})$. Задача $(\mathrm{C})$ - это задача нахождения напряжений и деформаций в упругой балке с модулями $E^{u}$ при заданной кривизне и нулевых остаточных деформациях. Её решение дают выражения

$$
\varepsilon=\kappa y, \quad \sigma^{\prime}=E^{u} \varepsilon, \quad M^{\prime}=I^{u} \kappa,
$$

где $M^{\prime}$ - изгибающий момент, отвечающий заданной кривизне $\kappa$ при изгибе упругой балки. Задача (D) - это задача об определении напряжений при известных остаточных деформациях и нулевой кривизне. Её решение можно получить, наложив на решение задачи (B) решение задачи об изгибе упругой балки с моментом обратного знака $\left(-M^{\phi}\right)$ таким, чтобы суммарная кривизна балки была равна нулю. В результате имеем

$$
\kappa=0, \quad e=0, \quad \sigma^{\prime \prime}=-E^{u} \varepsilon^{p}, \quad M^{\prime \prime}=-M^{\phi} .
$$

Пусть теперь при кривизне $\kappa_{0}$, которой отвечает момент $M_{0}$, балка находится в равновесии (см. начало п. 3). Возмутим это равновесие, увеличив 
кривизну на величину $\kappa_{\Delta}$. Параметры возмущения найдем, используя решение задачи (C) (формулы (15)). Имеем

$$
\varepsilon_{\Delta}=\kappa_{\Delta} y, \quad \sigma_{\Delta}^{\prime}=E_{0}^{u} \varepsilon_{\Delta}, \quad M_{\Delta}^{\prime}=I_{0}^{u} \kappa_{\Delta} .
$$

Тогда параметры балки в возмущённом состоянии равны $\kappa_{1}=\kappa_{0}+\kappa_{\Delta}, \varepsilon_{1}=$ $=\varepsilon_{0}+\varepsilon_{\Delta}, \sigma_{1}=\sigma_{0}+\sigma_{\Delta}^{\prime}, M=M_{0}+M_{\Delta}^{\prime}$ (см. рис. 3, 4). Далее, используя диаграмму деформирования, находим для значений $\varepsilon_{1}(y)$ распределение модулей $E_{1}^{u}(y)$ и по формуле (6) - распределение пластических деформаций $\varepsilon_{1}^{p}(y)$. Затем, применяя формулы (15) и (16), получаем решения задач (C) и (D), а именно

$\varepsilon_{1}=\kappa_{1} y, \quad \sigma_{1}^{\prime}=E_{1}^{u} \varepsilon_{1}, \quad M_{1}^{\prime}=I_{1}^{u} \kappa_{1}, \quad \kappa=0, \quad e=0, \quad \sigma_{1}^{\prime \prime}=-E_{1}^{u} \varepsilon_{1}^{p}, \quad M_{1}^{\prime \prime}=-M_{1}^{\phi}$.

Скорректированное напряжённо-деформированное состояние есть сумма решений задач $(\mathrm{C})$ и $(\mathrm{D})$, то есть

$$
\varepsilon_{2}=\varepsilon_{1}=\kappa_{1} y, \quad \sigma_{2}=\sigma_{1}^{\prime}+\sigma_{1}^{\prime \prime}, \quad M_{2}=M_{1}^{\prime}+M_{1}^{\prime \prime} .
$$

Так как деформации не изменились, то не меняются модули $E_{1}^{u}$ и пластические деформации $\varepsilon_{1}^{p}$. Следовательно, решение (17) окончательное. Схематически данная процедура показана на (рис. 3, 4) (прямая 2). Отметим, что используя этот итерационный процесс при постепенном возрастании величины $\kappa$, наряду с восходящей ветвью диаграммы $M \sim \kappa$ можно получить и нисходящую ветвь.

6. Упругопластический материал. Рассмотрим балку из упругопластического материала, который отличается от рассмотренного партипластического материала только тем, что имеет постоянный модуль разгрузки, равный модулю Юнга $E$. Поэтому при реализации построенных выше итерационных процессов во всех формулах п. $2, \mathbf{3}, \mathbf{5}$ следует величины $E_{i}^{u}$ и $I_{i}^{u}$ заменить соответственно значениями $E$ и $I=2 b h^{3} / 3$ и в формуле (6) положить $E^{u}=E$. Проводя затем рассуждения, аналогичные приведённым выше при исследовании итерационного процесса для мягкого нагружения, приходим к формуле (13), в которой $I^{u}=I$. Однако сходимость и расходимость итераций опять определяются знаком интеграла $I^{p}$, а начало расходимости и потеря устойчивости процесса изгиба происходит при обращении этого интеграла в нуль. При жёстком нагружении процесс сходится так же, как в п. $\mathbf{5}$.

7. Упругохрупкий материал. Пусть балка изготовлена теперь из упругохрупкого материала, в котором не образуются пластические (остаточные) деформации и модуль разгрузки равен секущему модулю. Тогда, используя формулы (8), находим, что

$$
M=2 b \int_{0}^{h} E^{s} \varepsilon y d y=2 b \kappa \int_{0}^{h} E^{s}(\kappa y) y^{2} d y=\kappa I^{s}(\kappa),
$$

где $I^{s}(\kappa)$ - жёсткость при изгибе упругой балки с модулями $E^{s}(\varepsilon)$. Отсюда решение задачи $(\mathrm{A})$ имеет вид

$$
\kappa^{\prime}=\frac{M}{I^{s}}, \quad \varepsilon^{\prime}=\kappa^{\prime} y, \quad \sigma^{\prime}=E^{s} \varepsilon^{\prime} .
$$


Решение же задачи (В), очевидно, равно нулю.

Возьмём некоторое положение равновесия $\left(M_{0}, \kappa_{0}\right)$ с параметрами $E^{s}=$ $=E_{0}^{s}\left(\varepsilon_{0}\right), \varepsilon=\varepsilon_{0}=\kappa_{0} y, \sigma=\sigma_{0}\left(\varepsilon_{0}\right) \in \sigma_{\varepsilon}$ и возмутим его, увеличив изгибающий момент на величину $M_{\Delta}$. По формулам (19) находим параметры возмущения, а именно

$$
\kappa_{\Delta}^{\prime}=M_{\Delta} / I_{0}^{s}, \quad \varepsilon_{\Delta}^{\prime}=\kappa_{\Delta}^{\prime} y, \quad \sigma_{\Delta}^{\prime}=E_{0}^{s} \varepsilon_{\Delta}^{\prime},
$$

где $I_{0}^{s}=I^{s}\left(\kappa_{0}\right)$. Тогда параметры балки в возмущённом состоянии равны

$$
\kappa_{1}=\kappa_{0}+\kappa_{\Delta}^{\prime}=M / I_{0}^{s}, \quad \varepsilon_{1}=\varepsilon_{0}+\varepsilon_{\Delta}^{\prime}=\kappa_{1} y, \quad \sigma_{1}=\sigma_{0}+\sigma_{\Delta}^{\prime},
$$

где $M=M_{0}+M_{\Delta}$. По диаграмме деформирования для значений $\varepsilon_{1}(y)$ определяем распределение секущих модулей $E_{1}^{s}(y)$ и решаем задачу $(\mathrm{A})$. Имеем

$$
\kappa_{2}=M / I_{1}^{s}, \quad \varepsilon_{2}=\kappa_{2} y, \quad \sigma_{2}=E_{1}^{s} \varepsilon_{2} .
$$

Здесь $I_{1}^{s}=I^{s}\left(\kappa_{1}\right)$ - жёсткость балки с модулями упругости $E_{1}^{s}\left(\kappa_{1} y\right)$. Для деформаций $\varepsilon_{2}(y)$ находим новое распределение модулей $E_{2}^{s}=E^{s}\left(\varepsilon_{2}\right)$ и опять решаем задачу (А). Процесс повторяем необходимое число раз. Схематически данная процедура для материального элемента показана на рис. 5 , а для балки в целом на рис. 4 .

На $n$-ном шаге итерационного процесса получаем равенство

$$
\kappa_{n+1}=M / I_{n}^{s}=\psi\left(\kappa_{n}\right) .
$$

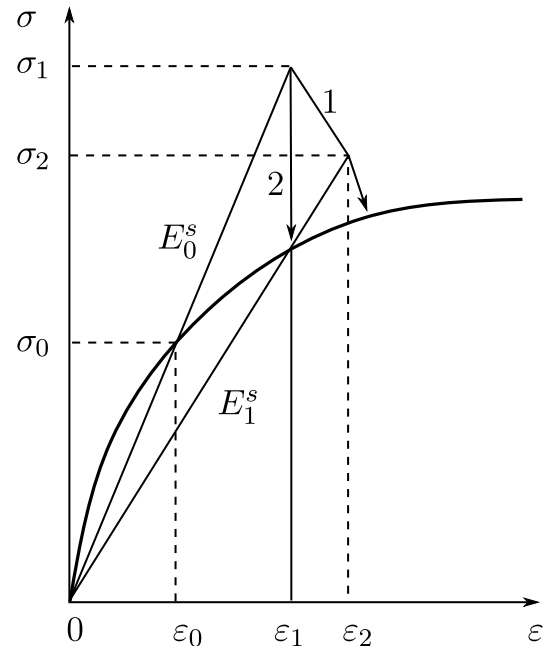

Рис. 5. Схемы итерационных процессов для элемента упругохрупкого материала: 1 -мягкое догружение, 2 -жёсткое догружение

Формула (20) определяет метод простой итерации. Для оценки его сходимости вычислим производную от функции $\psi(\kappa)=M / I^{s}$. В силу условия равновесия (18) имеем

$$
\frac{d \psi}{d \kappa}=\frac{-M \frac{d I^{s}}{d \kappa}}{\left(I^{s}\right)^{2}}=-\frac{\kappa I^{s} \frac{d I^{s}}{d \kappa}}{\left(I^{s}\right)^{2}}=-\frac{\kappa \frac{d I^{s}}{d \kappa}}{I^{s}} .
$$

Здесь

$$
\begin{gathered}
\frac{d I^{s}}{d \kappa}=2 b \int_{0}^{h} \frac{d E^{s}}{d \kappa} y^{2} d y, \quad \frac{d E^{s}}{d \kappa}=\frac{d E^{s}}{d \varepsilon} \frac{d \varepsilon}{d \kappa}=\frac{d E^{s}}{d \varepsilon} y \quad(\varepsilon=\kappa y) ; \\
\frac{d E^{s}}{d \varepsilon}=\frac{d}{d \varepsilon}\left(\frac{\sigma}{\varepsilon}\right)=\frac{\frac{d \sigma}{d \varepsilon} \varepsilon-\sigma}{\varepsilon^{2}}=\frac{E^{p}-E^{s}}{\varepsilon} .
\end{gathered}
$$

Подставляя эти выражения в формулу (21), после необходимых преобразований находим, что

$$
\frac{d \psi}{d \kappa}=1-\frac{2 b I^{p}}{I^{s}}
$$


Выражение (22) аналогично формуле (13). Таким образом, сходимость и расходимость, как и выше, определяются знаком интеграла $I^{p}$, а начало расходимости и момент потери устойчивости процесса нагружения балки совпадают с обращением интеграла $I^{p}$ в нуль.

При жёстком догружении итерационный процесс всегда сходится так же, как и в п. 5. Для элемента упругохрупкого материала этот процесс определяется прямой 2 (см. рис. 5).

Заключение.

1. Предложены итерационные процедуры расчёта напряжённо-деформированного состояния прямоугольных балок при чистом изгибе для различных типов материалов (партипластических, упругопластических и упругохрупких).

2. Показано, что сходимость этих процессов в условиях активного деформирования, который реализуется при чистом изгибе, не зависит от типа материала (вида разгрузки), а определяется только касательным модулем полной диаграммы деформирования.

3. Установлено, что начало расходимости итерационного процесса совпадает с моментом потери устойчивости процесса нагружения балок.

Работа выполнена при поддержке РФФИ (код проекта 10-08-00135).

\section{БИБЛИОГРАФИЧЕСКИЙ СПИСОК}

1. Стружанов В.В., Жижерин С. В. Модель повреждающегося материала и итерационные методы расчёта напряженного состояния при кручении // Вычислительные технологии, 2000. - Т. 5, № 2. - С. 92-104.

2. Жижерин C. В., Стружанов В.В. Итерационные методы и устойчивость в задаче о равномерном деформировании шара с центральной зоной из повреждающегося материала // Изв. РАН. МТТ, 2004. - № 2. - С. 144-225.

3. Колмогоров A.Н., Фомин С.В. Элементы теории функций и функционального анализа. - М.: Наука, 1972. - 496 с.

4. Gilmore R. Catastrophe Theory for Scientists and Engineers. - New York: Dover, 1993. 666 р.; русск. пер.: Гилмор Р. Прикладная теория катастроф: В 2-х книгах; Кн. 1. М.: Мир, 1984. - 350 с. 
MSC: 56U78, 74R99

\section{ITERATIVE PROCEDURES OF EQUILIBRIUM PARAMETERS ESTIMATION AND PROCESS STABILITY OF PURE BENDING OF BEAMS FROM SOFT AND BRITTLE WEAKENING MATERIALS}

\section{V. Struzhanov, E. A. Bahareva}

1 Institute of Teoretical Engineering of the Ural Branch of RAS, 620083, Yekaterinburg, Komsomolskaya st., 34

2 Ural State University,

620219, Yekaterinburg, Lenin st., 51

E-mails: stru@imach.uran.ru, bahareva.e.a@mail.ru

The article is concerned with iterative procedures of stressedly deformed state estimation of pure bendind of the beams with rectangular cross-section which are made of soft and brittle materials. The beams exhibit the strain weakening effect. The method convergence is studied and the interdependence of iterative divergence and loading stability is found.

Key words: pure bending, hard and soft loading, iterative procedure, convergence, stability, simple iteration.

Original article submitted 24/II/2010; revision submitted $17 / \mathrm{III} / 2010$.

Valeriy V. Struzhanov (Dr. Sci. (Phys. \& Math), Chief Research Scientist, Division of Machines Mechanics \& Technology. Elena A. Bahareva, Graduate Student, Dept. of Theoretical Mechanics. 